\title{
Rolling Bearing Fault Diagnosis Based on Physical Model and One-Class Support Vector Machine
}

\author{
Li Xiangyang and Chen Wanqiang \\ Power Equipment Research Institute Xian Aeronautical College, 259 West Second Ring, Xian, China \\ Correspondence should be addressed to Li Xiangyang; lxygyl@163.com
}

Received 8 October 2013; Accepted 9 December 2013; Published 14 April 2014

Academic Editors: A. Z. Sahin and A. Tounsi

Copyright (C) 2014 L. Xiangyang and C. Wanqiang. This is an open access article distributed under the Creative Commons Attribution License, which permits unrestricted use, distribution, and reproduction in any medium, provided the original work is properly cited.

\begin{abstract}
This paper aims at diagnosing the fault of rolling bearings and establishes the system of dynamics model with the consideration of rolling bearing with nonlinear bearing force, the radial clearance, and other nonlinear factors, using Runge-Kutla such as Hertzian elastic contactforce and internal radial clearance, which are solved by the Runge-Kutta method. Using simulated data of the normal state, a self-adaptive alarm method for bearing condition based on one-class support vector machine is proposed. Test samples were diagnosed with a recognition accuracy over $90 \%$. The present method is further applied to the vibration monitoring of rolling bearings. The alarms under the actual abnormal condition meet the demand of bearings monitoring.
\end{abstract}

\section{Introduction}

Rolling bearings are widely used in high-end CNC machine tools, aircraft engines, measuring instruments, and other valuable equipment. It plays a key role for the entire work of the host. Carrying out the bearing condition monitoring and fault diagnosis is important to ensure that the equipment is in good working condition. Since fault-prone of early rolling bearing easily exists in most of the bearing work cycle, and most of them are potential problem. Therefore, bearing failure abnormality diagnosis has been a research hotspot [1]. Patil makes a summary about the research Status of rolling bearing fault diagnosis. Compared to fault detection technology, the theory of rolling bearing fault modeling and fault mechanism is particularly inadequate [2]. In recent years, more and more scholars pay attention to the study of rolling bearing fault modeling. Cao established a complex dynamic model about aligning roller bearings, studying the problem of surface damage and preload and radial clearance [3]. Australian scholar Sawalhi [4] established time-varying nonlinear gear integration model, which simulated local spalling and damage fault of bearing.Rafsanjani et al. [5] established a theory of rolling bearings nonlinear dynamics model, given the mathematical description of the inner ring, outer ring, and rolling element of local damage. Patel et al. [6] established a dynamic model of the deep groove ball bearings, studied on the vibration response of the bearing inner ring and outer ring when it came to single-point and multipoint failure.

About anomaly identification bearing, which is combined with failure mechanism analysis and intelligent diagnosis, this paper first built a physical model of the rolling bearing normal state, and obtain the system response by numerical methods. By dimensionless indicators as characteristic quantities such as kurtosis and peak and building up the bearing diagnosis model based on a class support vector machine, the test bearing diagnosis results show the effectiveness of this method.

\section{Physical Model}

The structural vibration model of ball bearings can be developed for the rigid rotor system that with the outer ring fixed, the inner ring is equivalent to the concentrated mass. 


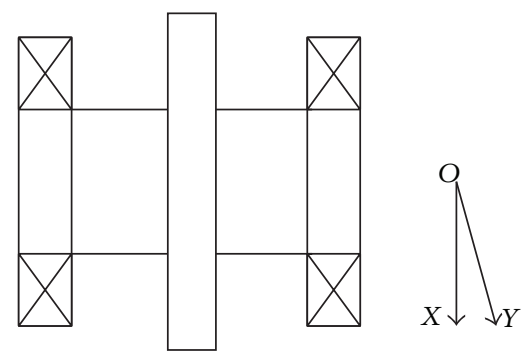

FIgURE 1: Ball bearing dynamics model.
TABLE 1: Ball bearings parameter table.

\begin{tabular}{lc}
\hline Bearings parameter & Numerical \\
\hline The bearing pitch circle diameter & $58.8 \mathrm{~mm}$ \\
Ball diameter & $11.9062 \mathrm{~mm}$ \\
Number of balls & 8 \\
Clearance & $40 \mathrm{um}$ \\
The quality of the rotor (inner ring) & $16 \mathrm{~kg}$ \\
External load & $58.8 \mathrm{~N}$ \\
Bearing damping & $2940 \mathrm{Ns} / \mathrm{m}$
\end{tabular}
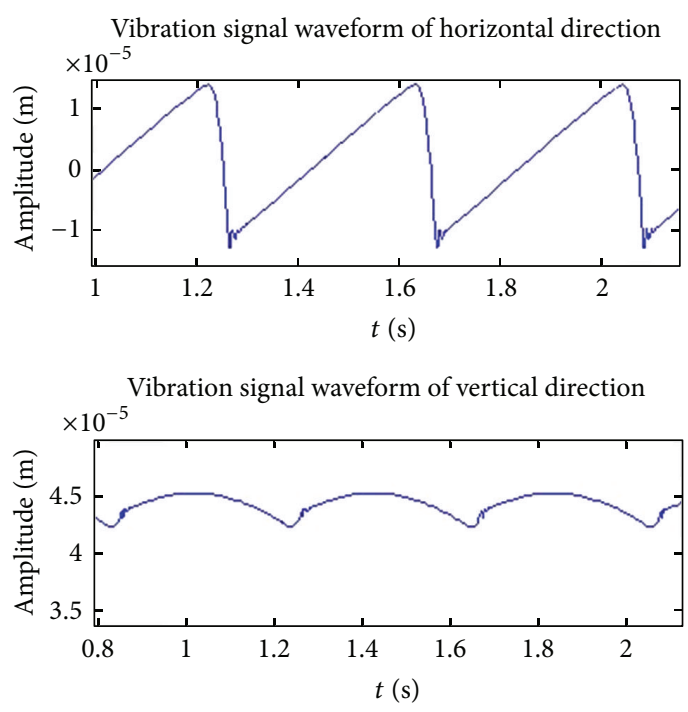

FIGURE 2: Time-domain waveform.

parameters [7]. Because formula (1) has a strong nonlinear state, it has no exact analytical results. This paper has got the system response using the Runge-Kutta numerical checking. Time-domain waveform is shown in Figure 2. The calculated results of FUKATA are shown in Figure 3. On the graph below, upper and lower diagrams, respectively, are bearing a vibration waveform of the horizontal and vertical directions. As seen from the drawing, the calculation results achieved good consistency. The amplitude of the minor difference is due to the material of the bearing and internal structure different parameters.

Therefore, coefficient of nonlinear deformation of point contacting is

$$
K_{b}=\frac{E}{\left(1-\left(1 / M^{2}\right)\right)} \sqrt{\frac{3 r_{\delta}}{(3 K / \Delta \mu)^{3} \sum \rho}} .
$$

The calculation methods of curvature about contact surfaces and $\sum \rho$ are given by literature [8]. Thus, once the size of the bearing material is determined, $K_{b}$ can be obtained by (2)-(4).

2.2. Reliability Verification of Model. In this paper, bearings used FUKATA is calculated in order to verify the correctness of ball bearing model and Table 1 gives the bearing

\section{One-Class Support Vector Machine}

One-class support vector machine is an unsupervised learning method based on statistical learning theory, having no prior knowledge, structural risk minimization, and so forth. Its purpose is to effectively distinguish the target class samples and other samples. The basic principle of one-class support vector machine is as follows [9]: assume that stems from the same distribution of $n$-dimensional vector samples $X_{1}, X_{2}, X_{3}, X_{4}, \ldots, X_{j} \in R^{n}$, samples are mapped to the high-dimensional feature space $F$ by nonlinear function. Look for a small size region that contains as many sample points in $F$, which make it separate with the nonclass sample 


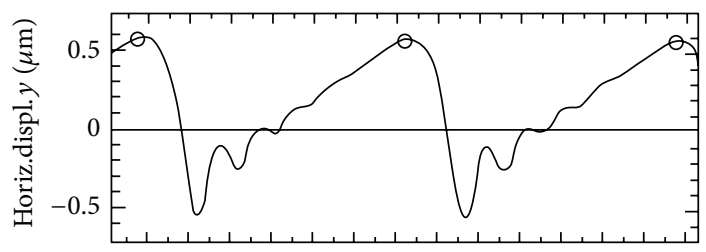

(a)

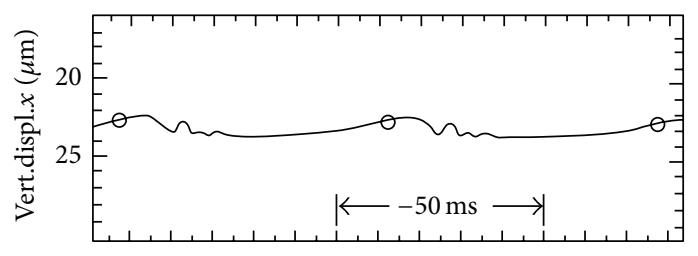

(b)

Figure 3: The calculated results of FUKATA.

at maximum intervals. I use the quadratic equation to represent the optimization problem. Quadratic type equation can be expressed as formula (5). In formula (5), $\omega$ is Hyperplane normal vector, $\rho$ is the boundary coefficient, $\xi_{i} \geq 0$ is relaxation factor, $l$ is sample points, and $0<v \leq 0$ is compromise coefficient that determines the distribution volume of the region that contains the sample points of compromise. From the point of view of,

$$
\begin{aligned}
& \min : \quad \frac{1}{2}\|\omega\|^{2}+\frac{1}{\nu l} \sum \xi_{i}-\rho, \\
& \text { s.t. } \quad\left(\omega \cdot \phi\left(x_{i}\right)\right) \geq \rho-\xi_{i},
\end{aligned}
$$

space geometry, sample is projected onto a hypersphere in the equation and the objective of this equation is to find a hyperplane that is quadrature and $\omega$. Its distance from the center of the sphere is $\rho /\|\omega\|$. The function of the equation, the main sample which is away from the center of the hypersphere distributed in this region and was split by hyperplane. In order to ensure the compact structure of the region, a few samples were excluded from outside the distribution area, with the distance from the boundary being $\xi_{i}$. Configuring Lagrangian function to solve formula (5), formula (5) is then simplified and we can get formula (6):

$$
\begin{array}{cl}
\min : & \frac{1}{2} \sum_{i, j=1}^{l} \alpha_{i} \alpha_{j} K\left(X_{i}, X_{j}\right), \\
\text { s.t. } \quad & \leq \alpha_{i} \leq \frac{1}{\nu l} \\
& \sum_{i=1}^{l} \alpha_{i}=1 .
\end{array}
$$

In formula (6), $\alpha_{i}$ is Lagrange coefficient, $K$ is Kernel function, and $K\left(X_{i}, X_{j}\right)$ is inner product calculation of $\phi\left(X_{i}\right)$ and $\phi\left(X_{j}\right)$ and is denoted $K_{i, j}$. Equation (2) is a typical quadratic programming problem. $\alpha_{i}$ obtained by solving majority is equal to 0 , and only a small portion of $A$ is greater than 0 . The sample corresponding to $\alpha_{i}$ is called a support vector. Decision function is defined as follows

$$
f(x)=\sum_{i=1}^{l} \alpha_{i} K\left(X_{i}, X_{j}\right)-\rho .
$$

In formula (7), when $f(x)>0, x$ is located in the distribution of samples within the area; when $f(x)=0, x$ is located at the edge of the distribution area; when $f(x)<0$, $x$ is located outside the distribution area of the sample. By formula (7), a sample of $\alpha_{i}=0$ is ignored in the calculation will. Therefore, the distribution of the target sample in the feature space of the whole only depends on the support vector.

\section{Diagnostic Methods of One-Class Support Vector Machine Based on Model}

The nature of the fault diagnosis is pattern recognition problem. In this paper, through numerical simulation to build a normal state of the sample, thus eliminate the problem by failure data. However the kurtosis, waveform, margin, peak and skewness of five dimensionless index [8] are the most important factors to a bearing failure, thus take advantage of the dimensionless indicators eigenvectors training oneclass support vector machine. Thus, I complete a bearing fault diagnosis. The process of this method is shown in Figure 4.

\section{Experimental Study}

An experimental study of the vibrated signal in the bearing test, which used to judge the bearing working state. Using SonyEX data acquisition system to get the signal, the sampling frequency is $10 \mathrm{kHz}$. Figure 5 is a typical test signal time-domain waveform and power spectrum. Table 2 is the anomaly identification result of one-class support vector machine. We can see that correct rate above $90 \%$ in Figure 5 and Table 2, which meet the demand of engineering. 


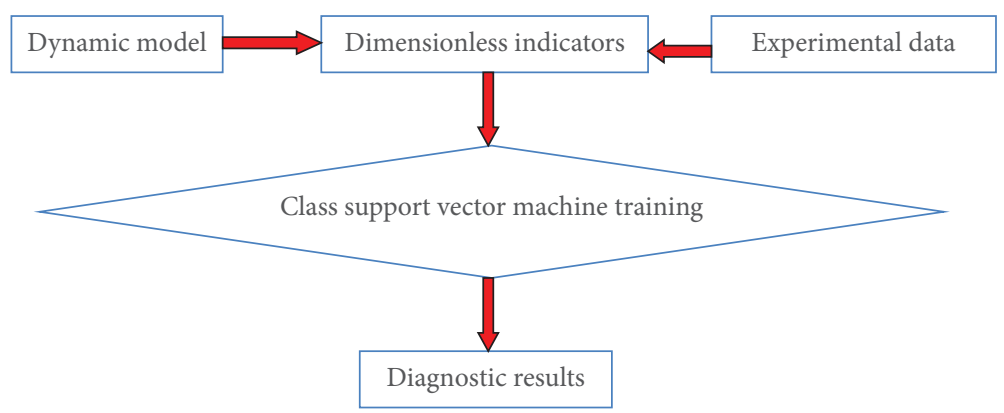

FIgURE 4: Class support vector machine diagnosis flowchart.
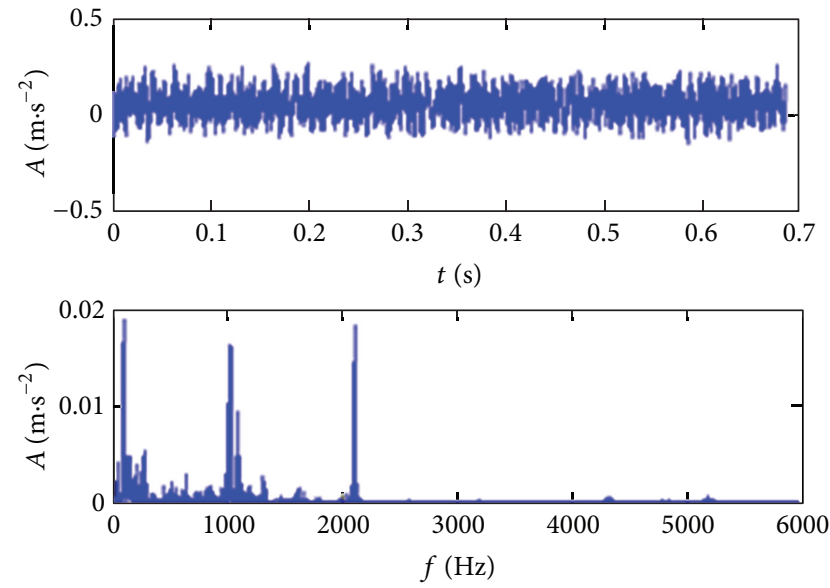

FIGURE 5: Normal signal waveform and power spectra.

TABLE 2: Abnormal rate of diagnosis of each state.

\begin{tabular}{lc}
\hline Bearing condition & Correct rate \\
\hline Inner ring defect & $94.3 \%$ \\
Outer ring defect & $90.6 \%$ \\
Ball defects & $91.7 \%$ \\
\hline
\end{tabular}

\section{Conclusion}

This paper presents a model-based ball bearing fault diagnosis method. It is through the use of dynamic models to get the timing of data reconstruction, thus taking advantage of the dimensionless indicators eigenvectors training one-class support vector machine to discover abnormal state. Engineering experiments show the feasibility and effectiveness of this method with the diagnosis rate being up to more than $90 \%$. This results meet the actual monitoring needs of the industrial field.

\section{Acknowledgment}

The authors would like to thank the Research Project of National Torch Plan in 2012 (2010GH041809).

\section{References}

[1] R. B. Randall and J. Antoni, "Rolling element bearing diagnos;atics-A tutorial," Mechanical Systems and Signal Processing, vol. 25, no. 2, pp. 485-520, 2011.

[2] M. S. Patil, J. Mathew, and P. K. R. Kumar, "Bearing signature analysis as a medium for fault detection: a review," Journal of Tribology, vol. 130, no. 1, Article ID 014001, 2008.

[3] M. Cao and J. Xiao, "A comprehensive dynamic model of double-row spherical roller bearing-model development and case studies on surface defects, preloads, and radial clearance," Mechanical Systems and Signal Processing, vol. 22, no. 2, pp. 467489, 2008.

[4] N. Sawalhi and R. B. Randall, "Simulating gear and bearing interactions in the presence of faults. Part I. The combined gear bearing dynamic model and the simulation of localised bearing faults," Mechanical Systems and Signal Processing, vol. 22, no. 8, pp. 1924-1951, 2008.

[5] A. Rafsanjani, S. Abbasion, A. Farshidianfar, and H. Moeenfard, "Nonlinear dynamic modeling of surface defects in rolling element bearing systems," Journal of Sound and Vibration, vol. 319, no. 3-5, pp. 1150-1174, 2009.

[6] V. N. Patel, N. Tandon, and R. K. Pandey, "A dynamic model for vibration studies of deep groove ball bearings considering single and multiple defects in races," Journal of Tribology, vol. 132, no. 4, Article ID 041101, 2010.

[7] S. Fukata, E. H. Gad, T. Kondou, T. Ayabe, and H. Tamura, "On the radial vibration of ball bearings (computer simulation)," Bulletin of the JSME, vol. 28, no. 239, pp. 899-904, 1985.

[8] T. A. Harris, Rolling Bearing Analysis, John Wiley \& Sons, New York, NY, USA, 1991.

[9] L. M. Manevitz and M. Yousef, "One-class SVMs for document classification," Journal of Machine Learning Research, vol. 2, pp. 139-154, 2001.

\section{Conflict of Interests}

The authors declare that there is no conflict of interests. 

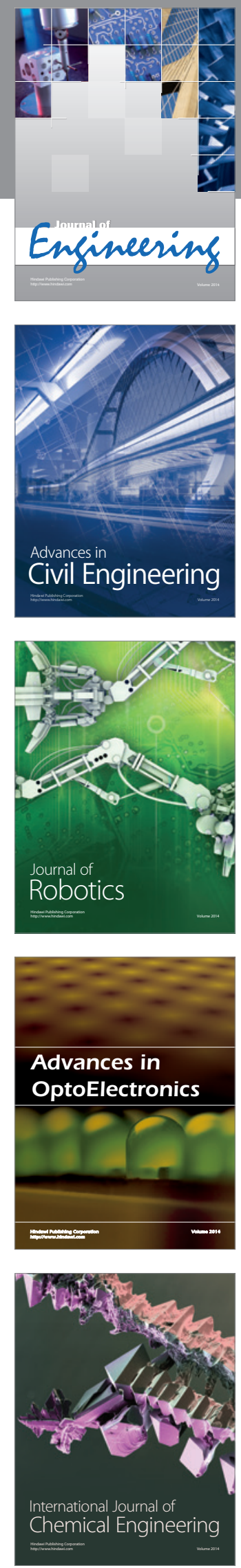

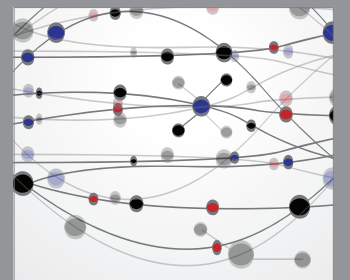

The Scientific World Journal
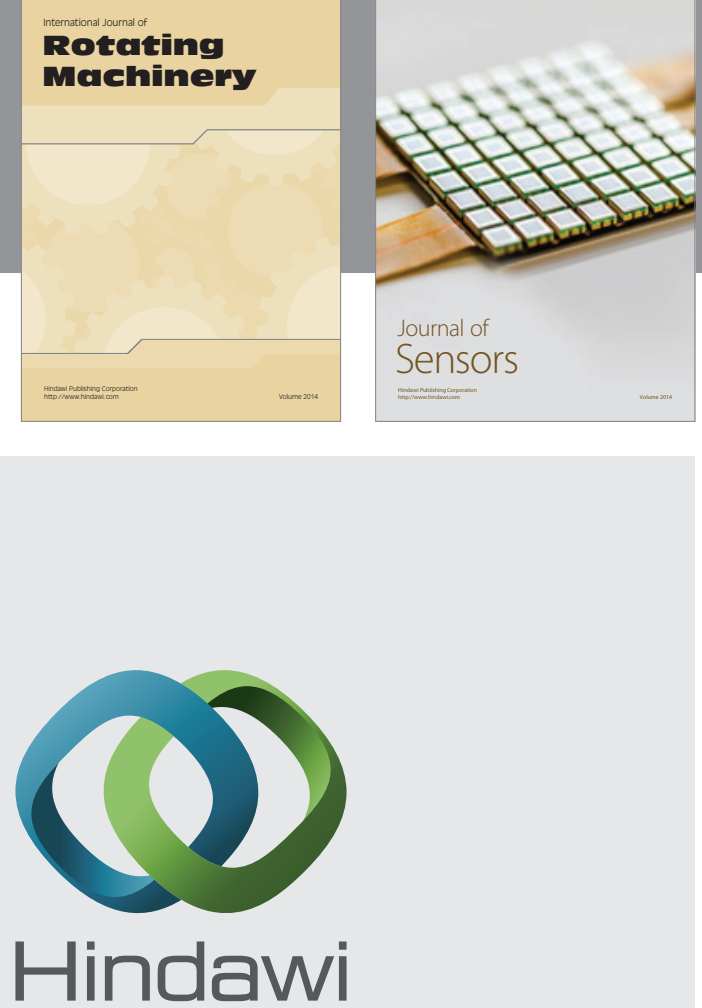

Submit your manuscripts at http://www.hindawi.com
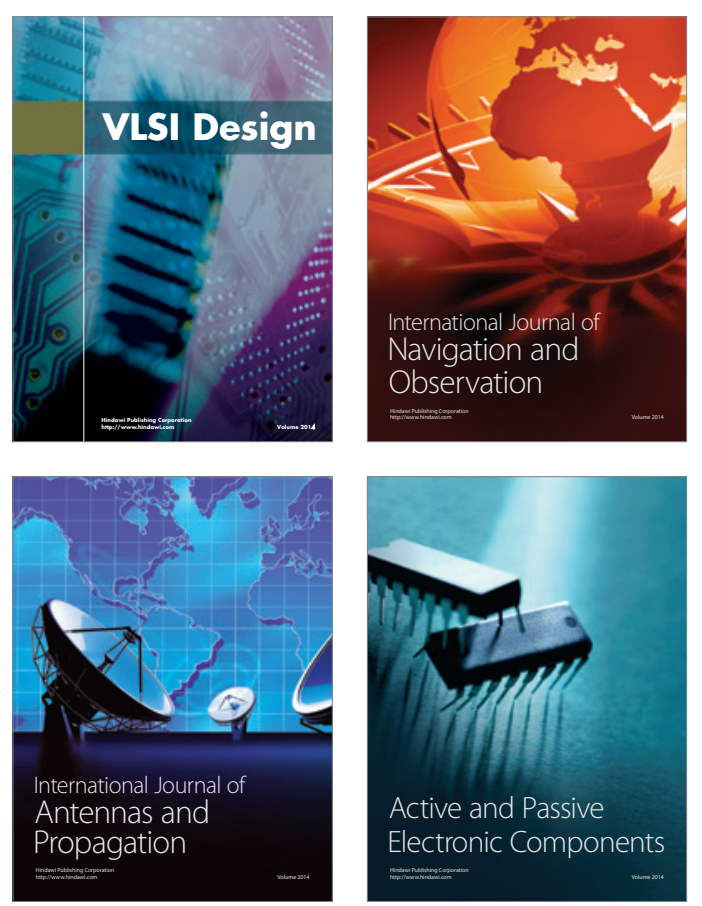
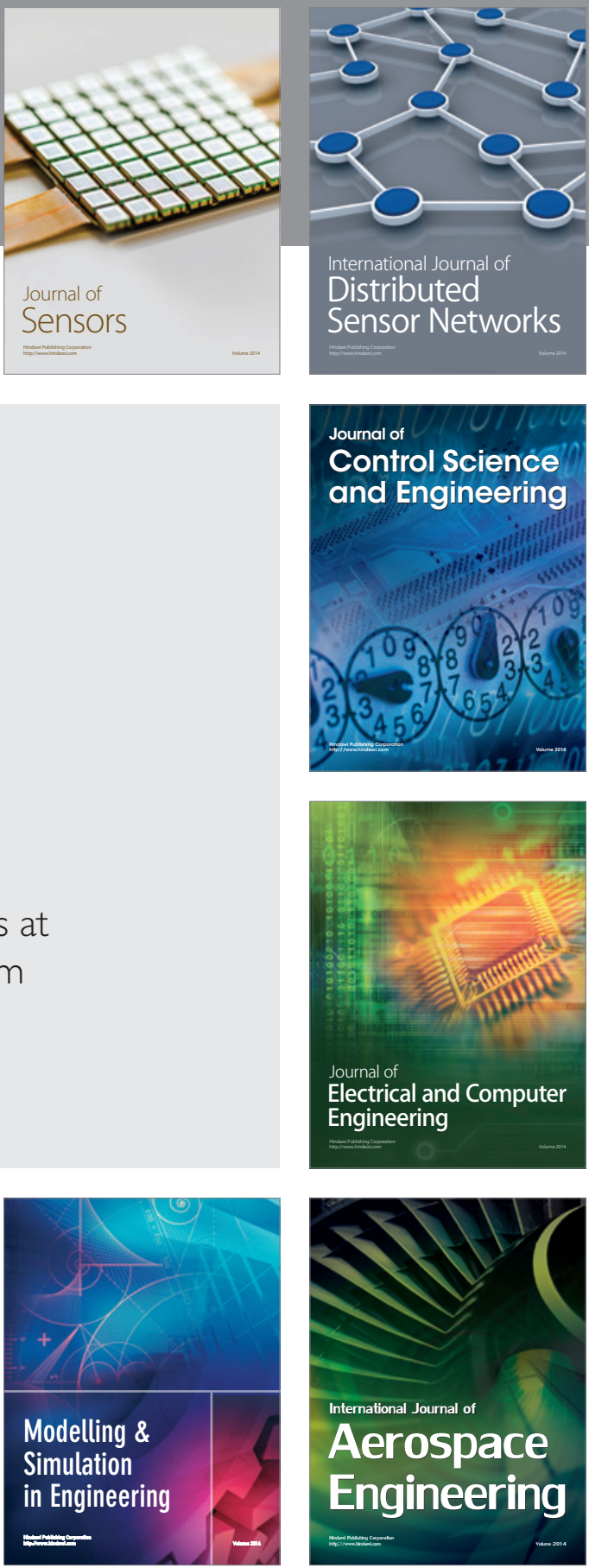

Journal of

Control Science

and Engineering
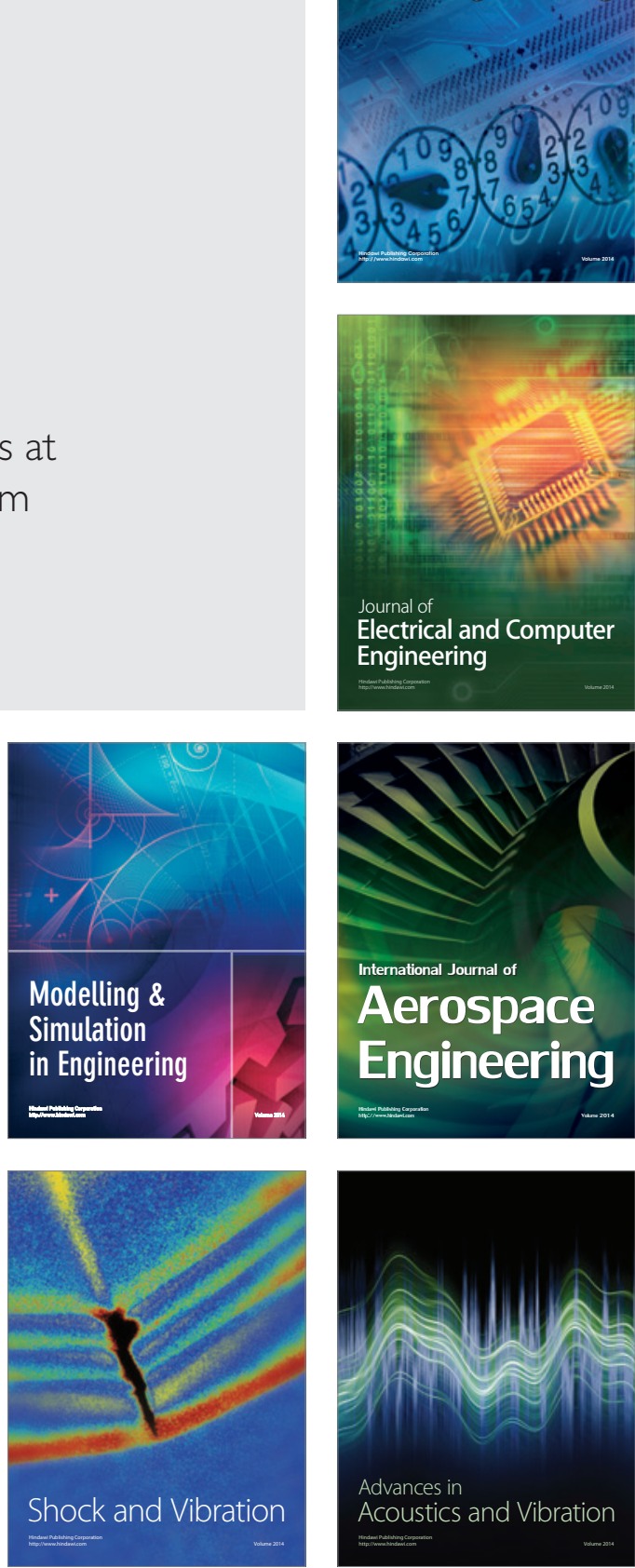